\title{
The PLATO space mission: studying planetary transits and stellar oscillations simultaneously
}

\author{
Malcolm Fridlund \\ Research and Scientific Support Department, ESTEC \\ Postbus 299, NL-2200AG, Noordwijk, the Netherlands \\ email: malcolm.fridlund@esa.int
}

\begin{abstract}
PLATO (PLAnetary Transits and Oscilliations of stars ) is a proposed mission of the European Space Agency's Science programme Cosmic Vision 2015-2025, currently under industrial study, and with a planned launch by the end of 2017. Its task is to better understand the properties of exoplanetary systems. As such it will detect and characterise exoplanets using their transit signature in front of a large sample of bright stars and simultaneously measuring the seismic oscillations of the parent star of these exoplanets. The mission will be orbiting the Sun-Earth L2 point, which provides a stable thermal environment and maximum uninterrupted observing efficiency. The payload consists of a number $(>28)$ of individual catadioptric telescopes, covering $>550$ sq. degrees. Since the goal is to search for terrestrial exoplanets within the habitable zone of their host stars, and carry out asteroseismological observations of the host stars, very high photometric precision, high time resolution, and high duty-cycle visible photometry is required. Ground-based observations are needed to complement the observations made by PLATO to allow for further exoplanetary characterization. This paper consists of a summary of the preliminary results achieved by the ESA internal pre-assessment study.
\end{abstract}

\section{Introduction}

The ESA Cosmic Vision Plan addresses four main themes through a number of future ESA science missions. These will be selected through an open competitive process involving the science community and within ESA Science Directorate programmatic constraints. The Cosmic Vision 2015-2025 (CV15-25) first Call for Mission Proposals was issued by ESA in March 2007 and aimed at defining one Medium-class mission and one Large-class mission for launch in 2017 and 2018 respectively. Following the CV15-25 proposal selection process, PLATO has been selected as an M-class mission candidate, for a further assessment study to be completed by Q3/2009. The readiness for launch by 2017 is a challenging demand, which in practice requires designing the space segment without new basic technology developments and with minimum developments risks. An internal pre-assessment study has been carried out as the first step in the assessment phase and is now being followed by two parallel industrial studies, each having a 12 month duration.

\section{Scientific Objectives}

The theory of planetary formation and evolution is very important in order to better understand the origin of life, and to determine whether and where life would be likely to exist elsewhere in the Galaxy. Likewise, the understanding of stellar internal structure and evolution is necessary in order to fully understand planets (e.g by determining stellar 
ages and by understanding the chemical evolution of stars). The evolution of planets and their central stars are linked, as they share a common history in terms of formation and early evolution. Stars affect the evolution of the orbiting planets, and the planets can themselves impact the evolution of the stars. Studying stars and their planets jointly is therefore necessary to understand the evolution of exoplanetary systems. These elements are for the first time, simultaneously addressed by the PLATO mission. The prime objectives of the PLATO mission are to search for exoplanetary transits (occultation) in front of stars and to characterize the parent star in terms of fundamental physical parameters via asteroseismology of the hosting star. PLATO will achieve this by high time-resolution, high precision, and high duty-cycle visible photometry and through complementary observations on-ground using e.g. high resolution spectroscopy. The asteroseismic data from PLATO will be needed in order to measure the stellar masses and ages. Using data from ESAs previous Gaia mission, allowing the determination of the stellar radius, the asteroseismological data obtained from PLATO will allow studying the internal structure, the internal angular momentum and the age of planet hosting stars with an unprecedented level of accuracy.

\section{Instrumentation and performance}

The PLATO telescopes are based on a catadioptric design with two SIC mirrors and two corrector plates. The optical design proposed suffers from a distortion of the FoV, meaning that the projection is not square but rather a distorted trapezoid. Since the observation strategy requires the spacecraft to rotate around the line-of-sight every three months to allow sufficient sun light to illuminate the solar arrays, not all the pixels in the CCD will see the same part of the sky for the entire duration observation of a sky field. Thus the science FoV is smaller than the instantaneous FoV. The science FoV is defined as the part of the observed sky field that is continuously visible on the CCD after a 90 degrees rotation around the line-of-sight. The achieved science FoV in the CDF baseline design is around 550 square degrees. At least 28 telescopes of which 26 will be aimed towards the main scientific objectives will be deployed, and the larger part of the spacecraft will consist of the optical bench on which the telescopes are mounted. At least 2 separate telescopes will perform an independent survey of very bright $\left(A_{V}<9\right)$ stars, as well as serving as startrackers. Each telescope will be equipped with a focal plane detector assembly consisting of a number of CCD's. Based on a $18 \mu$ pixel, each element CCD will have $3.5 \mathrm{k}$ by $3.5 \mathrm{k}$ active pixels (plus overclocking pixels). The CCD's to be used does not exist today and will have to be developed. PLATO will be able to observe half a million stars, in which it will be possible to detect planets the size of our Earth or smaller. Further, the large FOV will allow for 100000 of these stars to be so bright, that photometry down to the level required for accurate asteroseismology to be performed $\left(2.7 \times 10^{-5} h^{-1}-\right.$ for the larger sample the requirement is $\left.8.5 \times 10^{-5} h^{-1}\right)$. Due to the large FOV, PLATO will outperform the currently flying (and highly succesful) CoRoT satellite with a factor of 200 for exo-planetary capabilities, and no less than 3 orders of magnitude when it comes to a comparison in the field of asteroseismology. In the case of the NASA satellite Kepler (to be launched in 2009), PLATO will outperform it by a factor of 30 (for a given magnitude level) when dealing with planetary transits. The difference in performance for asteroseismology is no less than a factor 200.

PLATO, being launched 5-10 years after the pioneering missions, will be the first observatory to carry out large volumes of data acquisition at very high precision in the individual measurement. This will allow for a true 'union' of exoplanetology and stellar physics. 


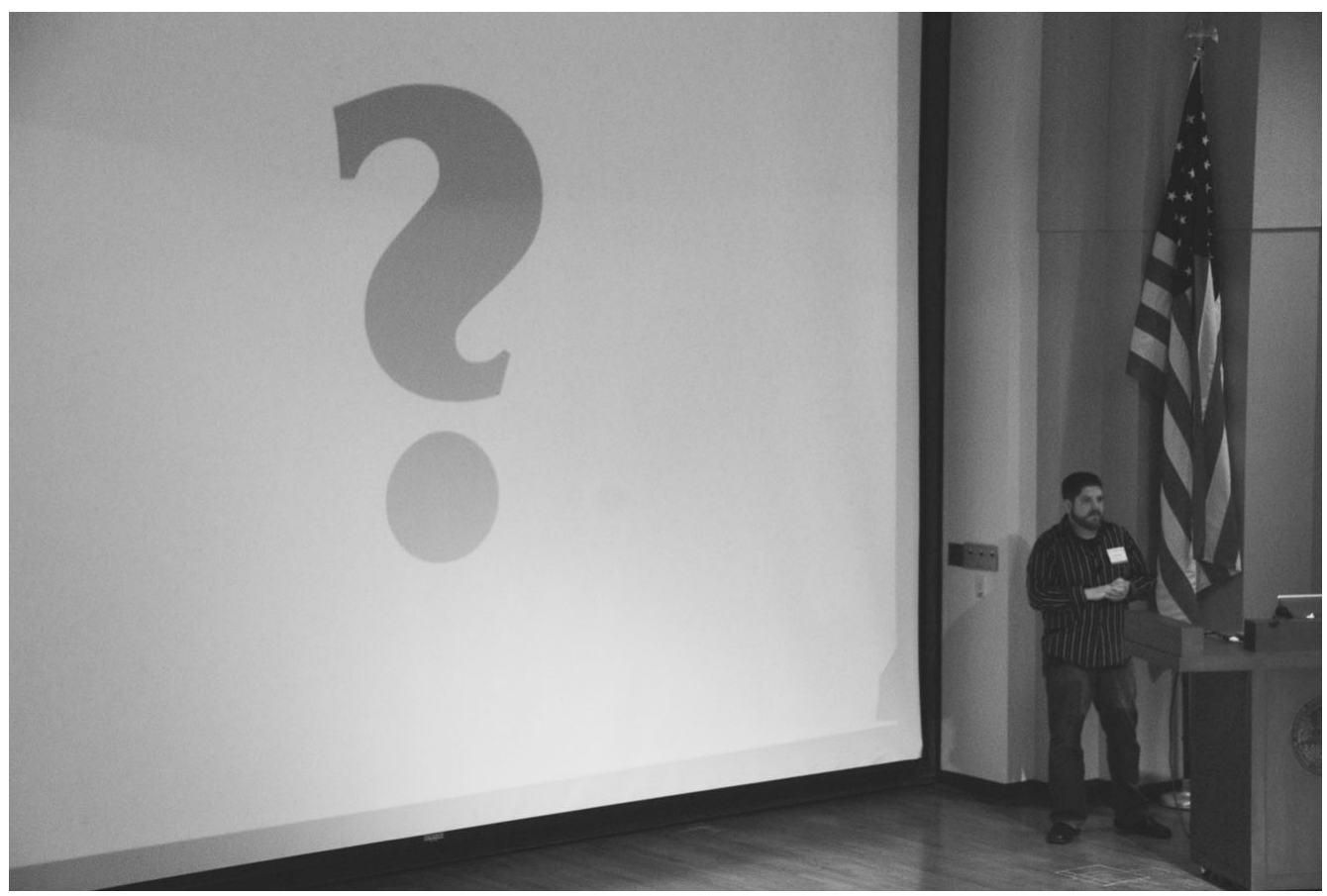

